\title{
Filigrane
}

Écoutes psychothérapiques

\section{Une étude des relations significatives d'un ex-détenu : aux sources identificatoires de la conflictualité}

\section{France Gabrion et Louis Brunet}

Volume 23, numéro 1, printemps 2014

URI : https://id.erudit.org/iderudit/1026081ar

DOI : https://doi.org/10.7202/1026081ar

Aller au sommaire du numéro

\section{Éditeur(s)}

Revue Santé mentale au Québec

ISSN

1192-1412 (imprimé)

1911-4656 (numérique)

Découvrir la revue

Citer cet article

Gabrion, F. \& Brunet, L. (2014). Une étude des relations significatives d'un ex-détenu : aux sources identificatoires de la conflictualité. Filigrane, 23(1), 115-135. https://doi.org/10.7202/1026081ar
Résumé de l'article

Cet article présente une analyse des relations marquantes ayant teinté la vie d'un ex-détenu dont la carrière criminelle a été caractérisée par de nombreux recels. L'objectif principal est de comprendre comment certaines relations significatives ont influencé le cheminement criminel d'un homme condamné à de multiples sentences d'emprisonnement. Douze entretiens avec un criminel réhabilité ont permis de dégager trois sphères conflictuelles de ses relations interpersonnelles, regroupées sous les thèmes suivants : l'importance de la méfiance, la protection contre l'attachement et la non-valeur d'autrui. Ces thèmes sont analysés et mis en rapport avec des figures significatives de son histoire, mettant en lumière la complexité des enjeux identificatoires et projectifs en cause. En outre, l'analyse fait ressortir comment des relations significatives sont devenues des identifications structurant le devenir criminel de cet homme. Le texte fait le lien entre ces analyses et les modèles psychocriminologiques et psychanalytiques des relations d'objet et des identifications. 


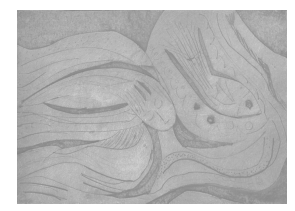

\section{Une étude des relations significatives d'un ex-détenu: aux sources identificatoires de la conflictualité}

\section{France Gabrion et Louis Brunet}

Cet article présente une analyse des relations marquantes ayant teinté la vie d'un ex-détenu dont la carrière criminelle a été caractérisée par de nombreux recels. L'objectif principal est de comprendre comment certaines relations significatives ont influencé le cheminement criminel d'un homme condamné à de multiples sentences d'emprisonnement. Douze entretiens avec un criminel réhabilité ont permis de dégager trois sphères conflictuelles de ses relations interpersonnelles, regroupées sous les thèmes suivants: I'importance de la méfiance, la protection contre l'attachement et la non-valeur d'autrui. Ces thèmes sont analysés et mis en rapport avec des figures significatives de son histoire, mettant en lumière la complexité des enjeux identificatoires et projectifs en cause. En outre, I'analyse fait ressortir comment des relations significatives sont devenues des identifications structurant le devenir criminel de cet homme. Le texte fait le lien entre ces analyses et les modèles psychocriminologiques et psychanalytiques des relations d'objet et des identifications.

\section{Introduction}

Charles ${ }^{1}$ a dévié du chemin qui était tout tracé pour lui. L'existence de cet homme, aujourd'hui à l'aube de la soixantaine, débute de façon on ne peut plus banale: famille unie, valeurs positives, condition économique moyenne pour l'époque. Toutefois, à l'adolescence, sa vie prend un tournant dramatique. Charles commence alors à fréquenter des pairs déviants, qui stimulent chez lui une certaine tendance à la délinquance. Il est résolu à ne plus se conformer aux attentes, aux normes et à la morale de sa famille. Charles amorce dès lors une carrière criminelle ponctuée de bons coups mais aussi d'échecs et de multiples incarcérations, l'appât du gain étant omniprésent. Il a pour but de devenir millionnaire et il met tout en œuvre dans l'espoir 
d'atteindre cet objectif ultime. Par contre, jamais il ne parvient à réaliser ce rêve, et il en paie chèrement le prix : toutes ces années d'ombre, emmuré dans les pénitenciers québécois.

$\mathrm{Au}$ moment de nos entretiens avec lui, Charles se trouve toujours sous l'autorité de la justice, en libération conditionnelle totale. Il vit de l'aide sociale et de ses nombreuses conférences portant sur son vécu de criminel. Il collabore également à des documentaires, des films, des scénarios portant sur la criminalité. Dans un sens, nous pourrions dire que son passé de criminalité contribue aujourd'hui à son mode personnel de réinsertion sociale. Il vit de son histoire et des récits qu'il peut en faire.

Ce texte analyse le vécu subjectif de Charles dans le but de comprendre comment des relations personnelles significatives ont influencé sa personnalité et son parcours (d'abord criminel, ensuite dans le processus de désistement).

Plus spécifiquement, à partir de l'analyse de douze entretiens, nous tentons de comprendre comment certaines relations significatives peuvent avoir influencé consciemment et inconsciemment Charles dans son cheminement vers la criminalité, dans l'organisation de sa dynamique psychique expliquant le devenir criminel, ainsi que dans le désistement de celui-ci. Quelles sont les relations significatives de Charles, qu'en a-t-il intégré? Comment ces intériorisations ont-elles contribué à construire une structure de personnalité favorisant la criminalité ? De quelle façon de telles relations significatives peuvent avoir contribué à une modification dynamique de sa trajectoire criminelle ou de sa structure psychique?

Le texte qui suit présente d'abord quelques modèles théoriques des relations d'objets. Par la suite, la méthodologie employée dans la présente étude ainsi que les objectifs visés sont présentés. Nous verrons enfin ce que l'histoire de Charles nous a appris et ce qu'elle nous permet de dégager comme compréhension des enjeux relationnels en cause.

\section{Les identifications dans la psychocriminologie psychanalytique}

Les identifications sont fondamentales dans le développement des structures de la personnalité, telles que le Surmoi, le Moi ou encore le Moi idéal (surtout dans la délinquance). Au-delà des théorisations de Freud (1900, 1923) sur le rôle de l'identification dans la structuration du Moi et du Surmoi, de nombreux auteurs ont noté l'importance des vicissitudes de l'identification dans ce qui a été considéré comme des «défauts » ou lacunes de l'organisation psychique des criminels. Ainsi, Aichorn (1925) y a vu l'origine de 
lacunes du Moi, Johnson et Szurek (1952) celle d'un Surmoi partiellement lacunaire, Mailloux (1971) la source d'une identité négative, et Kernberg (1975) la base même de pathologies du Surmoi dans une organisation morbide du narcissisme.

Cette vision d'une pathologie du Surmoi, si elle reflétait bien les observations cliniques, était cependant trop simple sur le plan dynamique et ne permettait pas de comprendre la complexité des multiples identifications, souvent contradictoires, en jeu chez les criminels. Suite à l'idée d'une différenciation théorique entre le Moi idéal et le Surmoi (Lagache, 1951; Lussier, 2006), divers auteurs ont mis en lumière comment certaines de ces identifications pouvaient s'intégrer dans le Moi idéal plutôt que dans le Surmoi, contribuant ainsi à une dynamique dans laquelle les interdits provenant du Surmoi étaient facilement désinvestis au profit de l'expansion narcissique que permet le Moi idéal (Brunet et Casoni, 2003; Casoni et Brunet, 2003, 2007). Ainsi, alors que les diverses formes identificatoires pouvaient sembler confuses dans la littérature psychanalytique, Lussier (1975), en se basant sur les écrits de Freud, a tenté de clarifier le tout en proposant des définitions du Moi idéal, de l'Idéal du Moi et du Surmoi. Casoni et Brunet $(2003,127)$ présentent brièvement en quoi consistent ces distinctions:

[en] suivant à la fois les points de vue dynamique, structural et économique, Lussier explique comment les identifications narcissiques, liées aux fantasmes de toute-puissance, structurent le Moi idéal; comment les identifications à des idéaux réalistes enrichissent le Moi et forment l'Idéal du Moi ; et enfin, comment d'autres identifications à des interdits forment le Surmoi dont la fonction est interdictrice et punitive par rapport au Moi.

À la lumière des principaux modèles psychanalytiques de la délinquance et de la criminalité, il ressort que trois séries d'identifications peuvent entrer en conflit dans la personnalité de celui qui devient criminel: celles s'inscrivant au sein du Moi, du Moi idéal et du Surmoi. À cet effet, à la suite des travaux de Lagache (1951), Casoni et Brunet (2003) expliquent que les identifications à l'agresseur du futur délinquant vont renforcer le Moi idéal plutôt que le Surmoi, ce qui oblige à considérer de quelles façons des identifications distinctes au même objet s'inscriront différemment dans les instances psychiques. Nous devons la notion d'identification à l'agresseur à Anna Freud (1949), qui la conçoit d'abord comme une étape du développement normal du Surmoi mais aussi comme un mécanisme de défense qui permet 
à l'enfant d'imiter l'agresseur (en acte ou en symbole) lorsqu'il est confronté à un danger externe. L'enfant n'adopte donc pas le rôle de la victime mais le renverse plutôt en position active. Afin de mieux comprendre comment peut s'instaurer chez l'enfant l'identification à un agresseur dans un contexte familial de maltraitance, par exemple, Sarnoff (1951) suppose qu'il y a trois préalables au développement de ce processus identificatoire. Il faut donc noter la présence:

- d'un agresseur qui veut imposer son autorité sur un tiers perçu négativement;

- d'une victime qui dépend de l'agresseur et qui ne peut échapper à son hostilité;

- d'une situation (sociale ou familiale) dans laquelle la victime se trouve globalement sous l'influence de l'agresseur.

Dans tous les cas, il va de soi que de s'identifier à un agresseur suppose d'ores et déjà un sujet probablement victimisé, dans une certaine mesure, ainsi qu'un individu malintentionné dont on suppose une mainmise quelconque sur la dite victime. Ce n'est pas là une évolution dite normale. Effectivement, il arrive que certaines identifications, au lieu d'aider l'enfant à faire face aux contenus pulsionnels, contribuent au développement de problématiques particulières.

Alors que les premiers modèles psychanalytiques de la délinquance ne distinguaient pas le Moi idéal de l'Idéal du Moi ou du Surmoi, les modèles contemporains ont tendance à voir dans le rapport Moi idéal/Surmoi une explication fondamentale de la possibilité de transgression des lois. Brunet et Casoni (2003) en font le centre d'une constellation dynamique dans laquelle l'identification à l'agresseur semble renforcer directement le Moi idéal, dont l'investissement croît tant à travers la recherche de la grandiosité et de l'omnipotence que le Surmoi devient complètement désavoué. Il ne s'agit plus de penser qu'il n'y a pas de Surmoi ou qu'il n'y a qu'un Surmoi lacunaire comme dans les premiers modèles, mais plutôt de voir comment le Surmoi est mis hors circuit par le surinvestissement de la structure narcissique que constitue le Moi idéal (Casoni et Brunet, 2003). Concernant ce rôle du Surmoi dans la délinquance, il ressort en outre trois modèles explicatifs. Le premier, soutenu entre autres par Anna Freud (1949), suppose une carence identificatoire du Surmoi et, dans une moindre mesure, du Moi, qui découle de l'absence d'intériorisation des interdits. Le second appuie l'idée d'une identification de type antisocial à un parent (Redl, 1945). Le dernier modèle réfère à une incapacité à s'identifier (Mailloux, 1965), ce qui consisterait en une 
défense permettant au sujet de se protéger contre la dépression (Casoni et Brunet, 2003).

Ces façons de concevoir comment de multiples identifications au même objet peuvent soutenir une conflictualité spécifique entre le Moi idéal et le Surmoi méritent d'être mises à l'épreuve de la recherche clinique, afin non seulement d'en vérifier la pertinence théorique, mais également d'en retracer l'évolution à travers le temps. Car si des identifications en jeune âge ont contribué à la création d'une organisation délinquante, ne serait-il pas possible que des identifications tardives puissent en modifier la trajectoire et l'issue?

\section{Entretiens et modes d'analyse}

Dans un premier temps, il importe de rappeler que cette recherche s'inscrit dans le cadre d'une étude de plus large envergure, intitulée «Les trajectoires de vie menant au désistement criminel : étude de l'histoire des détenus condamnés à une peine d'emprisonnement à perpétuité». L'objectif principal de cette étude, menée par Casoni, Brunet et Pelland (2009), consiste à :

Saisir les trajectoires de vie des détenus condamnés à une peine d'emprisonnement à perpétuité en libération conditionnelle, afin d'identifier, le cas échéant, les événements, les interactions, les points tournants et/ou les rationalisations qui influencent ces détenus à initialement cesser de commettre des délits et, subséquemment, à maintenir ce désistement criminel dans le temps, malgré les contraintes sociales, économiques et physiques auxquelles ils sont confrontés au cours de leur réinsertion sociale.

Dans ce contexte, le sujet a été recruté par l'un des auteurs, après lui avoir manifesté son intérêt à participer au projet. Une rémunération était octroyée afin de pallier les désagréments financiers liés aux déplacements. Ainsi, nous avons rencontré Charles à douze reprises, une fois par semaine, pour des entretiens de 90 à 120 minutes approximativement en s'inspirant du modèle de «récit de vie». Pour débuter, nous avons lancé la question suivante: «J'aimerais que vous me racontiez l'histoire de votre vie, dans l'ordre que vous le désirez, de votre petite enfance à aujourd'hui, afin que je puisse en connaître toutes les subtilités.» Par la suite, Charles était invité, librement, à se raconter, en cohérence avec la méthodologie choisie. De fait, les entrevues ont été réalisées selon une méthode associative, et des analyses qualitatives et séquentielles ont été effectuées entre chaque entretien. Dans ce contexte, nous comprenons le récit de vie à travers les principes d'entrevue de la théorie ancrée et de l'approche 
psychodynamique, qui recourent aux mêmes critères de validité : la saturation, la cohérence, la convergence et la parcimonie (Brunet, 2009). Dans un premier temps, le processus d'analyse de la «grounded theory» implique le questionnement systématique mais flexible des données; la théorie se construit à partir des résultats de recherche. Le paradigme psychanalytique, quant à lui, comporte un mode d'entrevue associatif qui inclut sept aspects (Kvale, 1999): l'étude de cas individuelle, la non-directivité de la méthode d'entrevue, l'interprétation du sens, la dimension temporelle, l'interaction humaine (et transférentielle), la pathologie comme thème d'investigation et, enfin, l'instigation d'un changement. Ces deux modèles non-dirigés et complémentaires se basent sur l'association libre et sur l'écoute des relations transférentielles et contretransférentielles. Puisque nous étudions un seul individu, les informations recueillies, précieuses et nombreuses, prennent leur source dans la subjectivité et la dynamique des processus psychiques (Pedinielli et Fernandez, 2009). L'étude de la subjectivité de la personne signifie que ce sont ses traits propres, sa perception des événements, sa vision du monde et le sens qu'elle octroie aux choses qui constituent la porte d'accès à la compréhension de celle-ci. En ce qui concerne l'analyse, tel que le souligne Bertaux (2010), nous y avons procédé de façon concomitante à ce qui était fait sur le «terrain ». Nous avons eu recours à l'analyse-retour et à l'analyse par consensus, toutes deux visant la validation de nos inférences. L'analyse-retour est une analyse effectuée entre chaque entretien. Elle permet, par un retour au sujet, non seulement une meilleure écoute et une meilleure sensibilité de la part de l'intervieweur, mais aussi la validation graduelle des inférences, au fur et à mesure des analyses (processus en «spirale», décrit par Brunet, 2009), alors que l'analyse par consensus signifie que chaque élément de chaque entretien fait l'objet d'une analyse et de déductions consensuelles entre deux chercheurs.

\section{Analyses}

L'analyse des entretiens avec Charles fait ressortir deux types de relations identificatoires marquantes - les figures paternelles et le soutien des identifications criminogènes -, de même que quatre enjeux dynamiques centraux issus des intériorisations conflictuelles de ces relations marquantes. Les trois premiers enjeux ont donné lieu à trois stratégies défensives sur le plan relationnel - la méfiance, la protection contre l'attachement et la non-valeur accordée à autrui - et le quatrième a consisté en un mode de relation spécifique, empreint à la fois d'identification et de projection - les relations à des hommes plus jeunes. 
Nous verrons donc en quoi les diverses relations objectales du passé ont influé sur le mode de vie de Charles. Notons ici que notre compréhension du passé du sujet ne se base que sur ce qu'il nous a raconté. Nos interprétations ne sont donc imputables qu'à son histoire de vie, telle qu'elle a émergé lors de nos entretiens. Nous tentons donc de rendre justice, de façon fidèle, à un récit personnel et intime.

\section{Des dynamiques relationnelles déterminantes}

Lorsque nous y regardons de près, l'analyse des entretiens avec Charles montre que certaines identifications ont eu des conséquences majeures sur les motivations inconscientes menant à ses agissements antisociaux. Il s'agit notamment du rapport à son père et des identifications conflictuelles qui en ont découlé, ainsi que des relations vécues avec certains criminels idéalisés.

\section{Les figures paternelles}

Du discours de Charles ressort l'importance de son père dans son organisation psychique. Cependant, son influence semble tout à fait paradoxale dans son devenir criminel. Dans un premier temps, nous remarquons une relation positive au père dans l'enfance. Charles affirme à quelques reprises avoir reçu de lui de bonnes valeurs, et également avoir bénéficié d'une jeunesse saine.

J'ai eu une criss de belle enfance dans le fond, je regarde ça en prison c'est pas la majorité du monde qui a eu une belle enfance, au contraire, la majorité a été «barouettée".

Ses propos laissent voir une certaine admiration pour son père, ainsi que ses qualités.

Mon père était orgueilleux, mon orgueil je sais de qui je la tiens. Il n'était pas riche mais le dimanche il allait à la messe il était bien habillé en habit pis ben chic pis il sentait bon, ben rasé $[\ldots]$. On n'était pas riche mais ma mère faisait des miracles dans la couture.

Des qualités ternies par contre par le pendant négatif de la pauvreté.

Mes parents ont toujours été présents pis j'ai quand même une bonne famille, j'ai eu des bonnes valeurs d'inculquées [...]. Dans ma jeunesse, je 
voulais faire missionnaire, mais avec les années, $\mathrm{j}$ 'ai vu mon père comment il travaillait fort pis dur [...] pis il comptait ses cents.

$\mathrm{La}$ « richesse» faisait défaut à la famille, jouissance que Charles cherchera d'ailleurs à atteindre toute sa vie. Malgré les qualités et les valeurs parentales qu'il relate, il a graduellement rejeté ce mode de vie, familial et modeste. Ces quelques extraits illustrent ce que l'ensemble des entrevues a montré. Charles a connu un père auquel il s'est identifié, de qui il a acquis des valeurs et des principes moraux. Cependant, comme nous le verrons, cette identification au père qui aurait pu soutenir une organisation psychique dans laquelle l'Idéal du Moi et le Surmoi régulent les conduites de façon habituelle, va se complexifier pour donner lieu à un conflit identificatoire aux conséquences importantes. À partir de l'adolescence, nous observons un retournement dans la perception qu'a Charles de son père. Charles voit peu à peu son père comme étant faible et décevant, notamment parce qu'il n'a pas beaucoup d'argent. Il se produit alors une nouvelle organisation identificatoire basée sur un conflit: ressembler à son père ou le rejeter pour ne pas s'y apparenter. Dynamiquement, Charles cherche à devenir le contraire de cette nouvelle perception de son père, donc être fort, puissant et riche. L'analyse des entrevues laisse croire que se produit alors une inversion de l'investissement du Surmoi et du Moi idéal correspondant à ce nouveau conflit. Peu à peu s'organisent en parallèle un rejet du père et un rejet du Surmoi et des valeurs de l'Idéal du Moi (correspondant aux identifications paternelles), de même qu'un surinvestissement du Moi idéal qui se nourrira de nouvelles identifications adolescentes.

«Pis là je me disais: «Aille, je peux pas, je ferai pas ça moi toute ma vie, compter mes cents comme mon père.» [...]. J'ai assez vu ma mère compter ses cents, ma mère pis mon père $[\ldots]$... je ne voulais pas faire pareil moi, «Je vais être riche pis je compterai pas mes cents comme mes parents.».

Il était hors de question, pour le jeune Charles, de suivre les pas de ses parents, ces derniers étant confrontés au décompte constant de leurs avoirs. Il généralise cette perception de médiocrité à la classe moyenne, celle qui travaille et pour laquelle la vie équivaut à «métro-boulot-dodo». Cette populace, il la méprise depuis son adolescence, extension du mépris dirigé contre son père. 
Me regarder dans le miroir en me faisant la barbe pis me dire : «Ouin hein [...] ils t'ont eu hein, tu y vas travailler aujourd'hui !» [...]. Je pouvais pas accepter ça, ben voyons.

Ben non, pas question, je ne deviendrai pas un mouton comme tout le monde. (entendre: comme mon père)

Sur la scène externe, une révolte contre l'autorité, tout d'abord représentée par le père et les religieux, puis s'étendant à toute autorité, vient accentuer le renversement identificatoire.

C'était tout le temps, tout le monde voulait que je fasse tout ce qu'eux autres veulent là $[. .$.$] . Là je disais: "Non je suis tanné, moi je vais faire ce que je$ veux, quand je veux. »

C'est ça, les frères [communauté religieuse] étaient sévères, mon père était sévère et tout le monde me disait quoi faire [...] pis un moment donné... je me suis mis à dire: "Wo là, je vais faire ce que je veux. »

Ces religieux qui lui ont enseigné ont tenté, tout comme le père, de tracer un chemin salutaire pour l'enfant qu'était Charles. Toutefois, un de ces hommes d'Église, le frère Jean, aura fortement amplifié la tendance antisociale de l'adolescent.

Le frère [Jean] avec sa flûte à bec. Son solfège. Pis il riait de moi pour toutes sortes [...] d'affaires pis il me dénigrait là.

En tout cas, je le voyais, pour moi il avait l'air d'une tapette, frère [Jean], pis il était tout le temps après moi, il me complexait assez que criss je pensais de le tuer.

Il me ridiculisait dans la classe là, pour certaines affaires, mettons que... des niaiseries là [...] pis j'étais orgueilleux pis susceptible [...] me faire ridiculiser devant tout le monde je le prenais pas là.

Ici apparaît la nature complexe et conflictuelle du rapport au père, et par déplacement, du rapport aux hommes en autorité. Charles se sent méprisé, dénigré par ces figures masculines. Mais la circularité de ce dédain est déterminée par le même conflit qui le relie à son géniteur. Il se sent méprisé, tout comme il méprise la faiblesse et la pauvreté de son père, la nature conflictuelle œdipienne se dévoilant dans la nature homosexuelle latente ( «tapette») du mépris ouvertement utilisé. Qui allait être l’homme, viril, 
puissant et riche? Ce devait être lui, Charles, alors que le religieux et le père devaient être faibles, pauvres et même efféminés. Il était inconcevable pour l'adolescent de respecter la volonté des autres, de s'y plier. Cela aurait été vécu soit comme une identification à un père faible, soit comme une soumission dégradante. Il lui était impossible d'accepter la faiblesse relative à la pauvreté, tel un signe d'impuissance et d'abnégation dans le cadre d'une identification à son père. Charles s'est alors mis à investir une image de lui tout à fait contraire au père «faible et pauvre» qu'il décrit.

Vingt-cinq ans je vais être rendu millionnaire et puis je me voyais pas autre chose que millionnaire [...], moi je compterai pas mes cents toute ma vie.

Nous verrons que, par l'investissement de cette image, c'est un sentiment de toute-puissance et de grandiosité qui s'installe en lui, dans le but de contreinvestir l'identification à un père vu comme faible.

Je n'ai pas peur du diable. Je n'ai pas peur de personne, personne va me faire peur. Je suis un humain pis il n'y a pas un humain qui va me faire peur. Même avoir 88 ans, il y a personne qui va m’impressionner.

En somme, Charles entre en conflit avec les premières identifications positives à son père depuis qu'il réalise que ce dernier n'est ni puissant, ni riche. Cette conflictualité interne le mène à investir le Moi idéal au détriment des identifications paternelles plus modérées, mais comprenant des valeurs morales conformes à la société dans l'Idéal du Moi et le Surmoi. Cette transformation dynamique semble mettre en conflit les premières identifications au père (notamment du Moi et du Surmoi) et celles à un père faible. Plutôt que de résoudre cet antagonisme lié à la désillusion tout en conservant les identifications initiales, il semble que Charles ait plutôt surinvesti le Moi idéal (identifications archaïques à un père puissant): il aura recours à de nouvelles identifications post-œdipiennes de nature antisociale, comme nous le verrons plus loin, afin de renforcer ce renversement dynamique, ouvrant ainsi la voie à la délinquance.

\section{Le soutien des identifications criminogènes}

Comment compenser la blessure narcissique d'une identification à un père qu'il juge faible et méprisable? Charles prendra la voie de l'idéalisation de figures puissantes, tels les motards ou Roberto, puis il s'identifiera à eux afin de retrouver son narcissisme perdu. 
$[\ldots]$ une gang de motards qu'il y avait $[\ldots]$ à l'époque $[\ldots]$ pis je trippais sur eux autres.

J'aurais tout fait pour eux autres, je m'identifiais à quelqu'un.

Il s'est donc développé une appartenance à une nouvelle "famille», ce qui a eu pour effet de compenser le manque d'appartenance à «sa» famille qu'il méprisait alors, dont les membres attendaient de lui un idéal qu'il ne souhaitait pas intégrer ni respecter.

Tu développes un sentiment d'appartenance un moment donné.

Un peu plus tard, une rencontre en prison vient changer la vie de Charles, s'il est possible de le dire ainsi. Roberto personnifiait la richesse, la réussite, la gloire, et il représentait ce qu'il voulait lui-même devenir et être. Il était celui par qui Charles pouvait retrouver un père archaïque puissant qu'il avait perdu en méprisant son père réel.

C'était un des gros bandits [...] pis il aimait ça avoir des jeunes avec lui, dans le fond c'était pour leur faire faire les mauvais coups à eux autres pis tout, mais c'était un professionnel du vol à main armée de banque, toute sa vie il a fait ça. Tous les petits voyous [...] rêvaient de voler avec lui parce que le gars avait des gros chars, des gros yachts, des «ski-doo », [...] grosse maison pis... Fait qu'il était plein d'argent pis là c'était comme une idole un peu pour moi...

Pour Charles, un criminel qui réussit parvenait à s'enrichir, mais aussi à s'imposer, à susciter la peur et l'admiration. La richesse égalait la toute-puissance, et la toute-puissance signifiait la gloire et la reconnaissance. Ainsi, après avoir fait ses premières armes dans le gangstérisme, il apprend à connaître Roberto, à partir duquel sa criminalité semble prendre son essor à travers son identification à celui-ci.

Mais [...] quand t'es jeune, des coups de tête là, les vols avec [Roberto], ça, ça a marqué ma carrière dans le banditisme là [...], beaucoup, ça pis mes rencontres avec les gunmen au vieux pen.

Bon, vols avec [Roberto], c'est là que tout a vraiment commencé, ma grosse criminalité là. Faire de l'argent pis toute. 
Lorsque nous le questionnons sur l'apport concret de Roberto dans son existence, dans son cheminement, Charles n'hésite pas:

Sa réputation. Juste le fait que le monde disait: « Aille le petit [...] est rendu avec [Roberto], il travaille avec [Roberto] !» Hé ciboire ça a fait monter mon nom $[. .$.$] , c'est comme un jeune pas connu qui commence à voler là$ pis il a plein de nerf mais il a jamais fait de quoi de gros là mais aujourd'hui «woup» il est avec [...] [un chef mafioso], c'est le bras droit de... Ben tu sais qu'il va faire de l'argent.

Psychologiquement, cette alliance sous-tend une identification s'installant dans le Moi idéal. Concrètement, cette association permet au jeune Charles, alors au début de la vingtaine, de s'imposer dans le centre-ville, d'en prendre le contrôle, et de s'extraire du groupe de motards, dès lors inutile : la toute-puissance, il la possédait désormais. Roberto lui avait permis non seulement de «se faire un nom» (identité reliée au Moi idéal), mais de devenir lui-même une figure criminelle respectable et respectée. En outre, nous apprenons aussi que la relation avec Roberto se développera en trois aspects: projectif d'abord, idolâtré ensuite, dévalorisé à la fin. Ici, nous constatons la difficulté du sujet à accorder de la valeur à une personne "plus grande » que lui. Mais, en même temps, cette relation peut sembler une répétition du lien conflictuel au père dans le passage de l'idéalisation au dénigrement.

[Roberto] lui j'ai trippé sur lui avant d'être un de ses jeunes pour apprendre à voler des banques pis des affaires de même là. Mais après que je l'ai ben connu pis toute, je me suis aperçu que j'étais ben au-dessus de lui, que c'était rien ça.

Nous le constatons dans la phrase précédente: la conflictualité de Charles, toujours prise dans l'opposition idéalisation-mépris, père-enfant, ne peut lui permettre de demeurer longtemps dans une position où il admire un tiers. Bien que renforçant son propre Moi idéal, la conflictualité anale, qui semble issue de la relation au père, se répète. Il doit alors à nouveau dénigrer l'objet pour demeurer le seul grand, fort et riche; le seul qui peut et doit être idéalisé. Le clivage non résolu fait en sorte que l'objet originellement magnifié doit ensuite être dénigré. Nous remarquons que le renversement initial de la relation au père n'a pas réglé la conflictualité qui se répète dans ces nouvelles relations: à la fois à travers la recherche d'un père idéalisé et puissant, 
et à la fois dans un nouveau retournement, laissant Charles seul dépositaire de la toute-puissance.

\section{Le mode relationnel privilégié et conséquent aux relations d'objet}

Outre ces grands criminels idéalisés, le discours de Charles laisse poindre peu de liens qui seraient significatifs, soit parce qu'il en parle rarement, soit parce qu'il s'en défend. Son discours donne l'impression que peu de personnes ont eu une influence sur lui, sur sa vie, et surtout sur son désistement criminel. Il apparaît presque que Charles ne serait jamais réellement parvenu à s'attacher à qui que ce soit. Nous constatons cependant que certaines personnes semblent avoir endossé une relative importance, plus ou moins assumée. Que pouvons-nous comprendre de cela? Veut-il se protéger, garder le contrôle, ou est-il incapable de relation profonde? Nous verrons que Charles semble apte à aimer, mais qu'il doit alors impérativement se protéger de certains enjeux relationnels.

\section{La méfiance}

Charles a découvert très tôt dans sa carrière criminelle qu'il devait se méfier, non seulement de ses ennemis, mais aussi de ses partenaires. De la façon dont il nous parle de ses relations, nous avons l'impression qu'il ne fait confiance à personne, ou très peu, et qu'il est extrêmement méfiant. Il présente sans cesse une image d'indépendance, qui semble agir tel un bouclier, une protection contre l'attachement, contre la relation à l'autre dans sa dimension de besoin ou de dépendance.

Je serais mal à l'aise de ça, à mon âge être pogné pour me fier sur quelqu'un pis toute, j'essaie de me démerder tout seul.

Charles dira donc ne pas vouloir compter sur qui que ce soit, mais avoir cependant tenté de faire confiance à quelques reprises, malheureusement avec des conséquences émotionnelles pénibles, laissant ainsi entrevoir des signes de blessures narcissiques.

J'ai essayé de me fier sur une couple de personne un moment donné pis je me suis retrouvé Gros-Jean comme devant.

En somme, Charles a appris à rester sur ses gardes, à ne pas nouer de relation mettant en jeu la loyauté et la dépendance. Néanmoins, au-delà des 
enjeux narcissiques qui lui sont propres, certaines de ces craintes pouvaient être réalistes: dans l'univers de la criminalité, il est risqué de trop faire confiance ou encore de nourrir une confiance aveugle... Le danger ultime étant qu'un tiers abuse de lui. En conséquence, il a toujours fonctionné selon la prémisse suivante: «J'attaque avant que l'autre ne le fasse».

\section{La protection contre l'attachement}

Pour Charles, l'attachement semble équivaloir à la perte de son indépendance et de son autonomie. Nous retrouvons donc une crainte de la dépendance, mais aussi, probablement et plus profondément, la peur de la perte et de l'abandon, ce qu'il ne pourrait toutefois jamais admettre. Il endosse une position défensive pour ne rien « devoir » à quiconque, et ne pas nourrir de relation de dépendance. Il se méfie des autres, oui, mais il craint également que ces autres puissent devenir significatifs à ses yeux et ainsi mettre en souffrance son mode de vie. Nous constatons l'idée d'une dilution de l'investissement d'objet, qui implique que personne ne peut prétendre être important à ses yeux, ce qui signifie, dans la dynamique précédemment analysée, avoir une emprise sur lui.

Malgré tout, Charles est vraisemblablement capable de s'attacher. Il ne faut pas penser qu'il présenterait une sorte «d'incapacité objectale»: nous pouvons plutôt y voir une forme de protection contre des enjeux relationnels infantiles. En effet, Charles nous montre que, n'eussent été ces enjeux et ces angoisses, il aurait souhaité pouvoir se lier affectivement à quelqu'un. Ainsi, il nous parle de deux personnes, entre autres, avec lesquelles il aurait bien voulu entretenir une relation intime, pas seulement éphémère mais durable. Toutefois, cela ne s'est avéré avec ni l'une, ni l'autre, sans doute pour les raisons citées plus haut.

Pis même souvent j'ai pensé, je me voyais avec, faire ma vie avec elle, je me voyais vraiment là $[\ldots]$.

Avec [Simon], avec [Simon] ça a passé un poil que esti je me serais marié avec $[\ldots]$. Je me serais marié avec ce gars-là.

De même, au début des entretiens, il paraît évident qu'il se protège de l'engagement relationnel avec l'intervieweuse. Mais peu à peu le lien d'attachement finit par poindre, notamment lors des dernières rencontres, alors qu'il nous semble devenir de plus en plus significatif à ses yeux, peut-être malgré lui. 
Il y a pas grand monde là dans ma vie, même dans ma famille pis dans mes proches, que j'ai confié des affaires autant que je te dis à toi. Je t'en dis des affaires là que j'ai jamais contées à personne...

C'est la première fois que je m'ouvre à quelqu'un autant qu'à toi. Oui, ça je voulais te le dire parce que c'est vraiment la première fois que... je sais pas si t'es consciente.

Il souhaite que nous reconnaissions notre chance à cet égard, mais aussi que nous soyons mise au fait que notre relation est devenue importante.

Quand tu parles pis que quelqu'un t'écoute, tu peux lui faire confiance, mais c'est une personne c'est certain que tu développes un lien [...].

Ainsi, malgré que l'attachement semble source d'angoisse au point où Charles a toujours voulu l'éviter, il appert qu'il soit tout de même capable de se lier à l'autre. Toutefois, nous ne pouvons savoir si cela est susceptible de véritablement perdurer. De fait, si nous prenons l'exemple de la relation dans le cadre de cette étude, Charles savait qu'il y aurait une fin, ce qui peut de surcroît susciter une plus grande propension à l'attachement, car limité dans le temps, celui-ci devient dès lors moins menaçant.

\section{La non-valeur accordée à autrui}

De nombreux enjeux narcissiques infiltrent donc les relations de Charles; qu'il s'agisse de la dichotomie valorisation-mépris dans sa double valence interne et externe, ou de l'enjeu relationnel lié au besoin ou à la dépendance. La conflictualité narcissique nécessite pour Charles la mise en place de stratégies de protection, de défense, qui se manifestent dans ses relations et dans sa vision des objets. Une de celles-ci, peut-être la plus importante, consiste à retirer toute valeur à l'objet. Dans la plupart des relations le moindrement significatives que Charles décrit, il s'assure de démontrer qu'il possède beaucoup plus de valeur que l'autre, qu'il le surpasse en tout point. Il refuse de paraître faible (compensation de l'identification à un père qui évoque la faiblesse) ou de laisser poindre une quelconque impuissance (comme celle qu'il vivait face au frère Jean ou encore celle qu'il attribuait à son géniteur). Encore une fois, à travers cette stratégie défensive qui disqualifie l'objet, il est difficile de ne pas voir la répétition des enjeux narcissiques infantiles entre Charles et son père, lequel est parfois faible, parfois puissant, tantôt riche ou pauvre; double inversion de l'idéalisation vers le dénigrement (son père et lui-même). 
Dans toute relation, même amoureuse, ce n'est donc plus l'attachement, la confiance ou la qualité de la relation qui compte, c'est malheureusement le pouvoir, la puissance, et même la domination anale. L'autre n'aura plus de valeur en soi comme stratégie pour résoudre les enjeux narcissiques. Il sera donc perçu comme un modeste partenaire provisoire.

Tu me prends quand je suis là... j'essayais de ne pas m'attacher trop parce que je le savais moi aussi quand je vais rentrer va falloir que je l'oublie j'ai pas le choix ça me donne rien de rêver à elle, je vais peut-être passer 15-20 en dedans.

Ou encore, l'autre sera simplement considéré comme une personne sexualisée à sa plus simple expression.

C'est là que j'ai eu ma première relation avec une femme. Je disais : «Câlisse c'était aussi bon qu'avec... [un homme] ", c'était vraiment le cul là, le cul pour le cul.

Ces propos étant tout de même déclamés avec humour, grâce à des illustrations concrètes.

Moi, c'est toujours de la viande fraîche.

Même être en amour pis toute hein, j'ai aimé des filles, des gars. Quand tu peux avoir une Cadillac pis une Lincoln c'est bien mieux que d'avoir juste une Cadillac ou juste une Lincoln.

Bien entendu, mépriser ou renverser la valeur de l'objet est une stratégie qui implique également le sujet. Une telle inversion sert autant à éviter les enjeux relationnels de dépendance (dénigrement de l'objet) qu'à renforcer son sentiment d'autosuffisance, et par là à compenser un profond sentiment d'incomplétude et de non-valeur. Dans ce contexte défensif, l'autre est souvent considéré comme un objet dont il peut se servir à des fins sexuelles ou criminelles, et rejeter lorsqu'il n'est plus utile. Par exemple, Charles décrira sa relation avec une jeune femme qu'il a grandement aimée mais pourtant, malgré cet amour, il ne pouvait s'empêcher de lui être infidèle.

Et puis vraiment là... on cliquait sur toute, sur le sexe, dans toute, les mêmes pensées $[\ldots]$ mais fallait je couraille pareil. 
Dans cette relation comme dans beaucoup d'autres, Charles semble à la fois éviter de s'attacher et faire en sorte que l'autre soit dénigré, sinon humilié. Il crée un mode relationnel qui est une protection contre toute proximité impliquant des émotions. Par contre, curieusement, certains liens semblent échapper à ces stratégies relationnelles: ces personnes, des hommes pour la plupart plus jeunes que lui, semblent être l'objet d'une relation projective dans laquelle le jeune homme aimé semble représenter une partie infantile de lui-même, comme nous le verrons dans la section suivante.

\section{Des exceptions... "les jeunes chums»}

Charles a eu plusieurs partenaires masculins, autant amoureux et sexuels que criminels. Clairement, certains d'entre eux étaient des hommes qu'il a aimés et aidés. Il a donc, souvent, joué le rôle d'un père (fort, fortuné, protecteur et adulé). L'analyse de ces relations amoureuses révèle que, sur le modèle de l'identification projective, le jeune homme qu'il aimait le représentait, enfant désiré d'un père admiré et fort, rôle qu'il assumait donc auprès de ce compagnon. L'identification projective lui permettait dynamiquement de faire d'une pierre deux coups en ce qui concerne ses besoins relationnels et amoureux. Par identification projective, il pouvait à la fois être le jeune, chéri d'une figure paternelle vigoureuse et vénérée, et le père, puissant et aimant. De surcroît, le recours à l'adjectif "petit », lorsqu'il parle de ses jeunes partenaires amoureux, vient renforcer cette idée de représentation du petit enfant dans la relation à ce père idéalisé.

J'avais toujours un jeune ou deux sous mon aile, je les protégeais pis les petits jeunes ils se tenaient avec moi [...].

Ainsi, les jeunes semblent fréquemment devenir des personnes significatives à base projective : il se peut que Charles leur donne ainsi ce qu'il aurait voulu qu'on lui donne (bénéfice par procuration), forme de réparation illusoire pour la conflictualité paternelle décrite précédemment.

Moi en prison ben souvent j'étais avec des petits gars là pis j'étais comme, je le réalise par après, j'étais comme leur père.

Les relations avec ses chums reflètent à la fois son désir d'être aux antipodes de son père, et de réparer les manques de son enfance. En effet, elles lui permettent d'incarner le père protecteur qu'il n'a pas eu et de chercher à satis- 
faire ses propres besoins infantiles projetés sur ses chums. Il parvient à donner à un autre ce qu'il aurait aimé avoir sur le plan narcissique en le faisant participer à sa propre puissance. Il insiste par ailleurs sur le bien-fondé de ses intentions.

Dans le fond là il y en a [des vieux de la vieille] que c'était pour le cul, il y en a que c'était juste parce qu'ils se sentaient comme un père [face aux plus jeunes], moi j'ai toujours aimé ça aider les jeunes même si j'avais rien avec eux autres.

Ainsi, nous avons l'impression que la relation nourrie avec ces « jeunes» dépassait le simple besoin sexuel bien compréhensible des hommes en prison, pour être également sous-tendue par le besoin d'une relation père-fils inversée grâce au processus d'identification projective, dans laquelle Charles trouvait un double profit : être aimé en tant que père puissant et être aimé par son père à travers la relation projective au « jeune ». Il s'agit de la façon dont il est parvenu à combler un vide, un manque au plan de l'identification à son père. S’il a renversé cette position, ceci ayant motivé l'émergence de sa criminalité, il a aussi utilisé le pendant positif de son père pour permettre à des plus jeunes que lui, probablement aussi démunis au plan identificatoire, de profiter d'une figure protectrice, puissante et aimante.

\section{Conclusion}

Cette étude ne peut qu'enrichir notre compréhension du vécu intrapsychique de l'individu délinquant. Évidemment, les relations significatives de chacun sont uniques, mais nous pouvons croire que certaines influencent plus que d'autres le devenir de l'adulte, ses choix et son mode de vie. Dans le passé, Charles a vécu des déceptions et des insuffisances au plan identificatoire. Nous ne saurons jamais si son père était réellement si faible, mais les entrevues ont montré que le mépris aurait remplacé l'idéalisation de celui-ci. Cette inversion a eu des effets dramatiques sur la structuration de sa personnalité, dès lors qu'il semble avoir rejeté tant le père que les identifications surmoïques paternelles, laissant le champ libre au surinvestissement du Moi idéal et aux nouvelles identifications à des hommes puissants mais délinquants. Pour contrer la blessure narcissique issue de l'identification à un père jugé faible et méprisable, Charles a adopté une position de révolte plutôt que de deuil de l'objet archaïque; il a renversé le positif en négatif. Diverses rencontres ont bien sûr contribué à ce qu'il se trace un chemin dans la criminalité... et quel chemin! 
Une vie teintée par la transgression des lois et de la morale, certes, mais aussi par de multiples incarcérations et un stress constant: celui de manquer d'argent, crainte qu'il a si souvent perçue chez ses parents. Toute son existence a été marquée par le partenariat, la méfiance et la superficialité relationnelle, mais aussi par une tentative de réparation ou de mise en forme de la relation qu'il aurait souhaité avoir avec son père.

Sans épuiser la complexité de la dynamique de Charles et les aléas des diverses rencontres qui l'ont marqué et influencé, il semble que la désillusion de l'image de son père a été traumatique (soit envahissante pour le sujet, et déterminante pour l'avenir) et a donné lieu à une réaction faite de renversements, dans lesquels il est devenu l'homme puissant et riche que son père n'était pas. Cela fut vraisemblablement appuyé par le surinvestissement du Moi idéal, au prix d'un amoindrissement de l'importance du Surmoi et des identifications surmoïques. Ces inversions ne se sont pas produites qu'avec son père, mais dans la recherche de nouveaux pères à l'image du Moi idéal, puis par la recherche de jeunes hommes avec lesquels il pouvait tenter de corriger la désillusion traumatisante de son passé. Sur les plans relationnel et amoureux, Charles était malheureusement prisonnier de ses enjeux narcissiques, ce qui l'obligeait à se protéger d'un amour dans lequel la confiance et la dépendance auraient pu occuper un espace sain. Nous remarquons plutôt un besoin anal de triompher, d'être le meilleur, et même d'humilier. Charles évite assez systématiquement l'attachement, la dépendance ou le fait d'être redevable à l'objet. Il s'en protège, mais il peut arriver pourtant qu'il se fasse prendre au jeu et réactive ainsi la relation infantile qu'il n'a pas réussi à contenir. Nous n'avons là qu'une parcelle de ce qui caractérise Charles, mais nous pouvons nous douter qu'il soit possible de découvrir des aspects semblables chez d'autres délinquants, ajoutant dès lors des éléments à notre compréhension du devenir criminel.

Sur le plan théorique, cette étude à cas unique soutient les travaux qui cherchent à différencier les fonctions du Moi idéal et du Surmoi. Elle supporte aussi la conception qui veut que des identifications à la même personne puissent s'inscrire dans des instances psychiques différentes et alors donner lieu à une conflictualité importante. Elle conforte enfin les modèles de la délinquance qui décrivent non seulement une relative faiblesse dynamique du Surmoi, mais surtout l'importance dynamique du Moi idéal dans le peu d'effet du Surmoi.

Malgré les limites de notre recherche, soit l'échantillon restreint à un seul individu ainsi que le choix de la méthodologie qui place en avant-plan la sub- 
jectivité du chercheur (malgré les procédés de contrôle par l'analyse par consensus), nous croyons que des études sur un seul sujet peuvent être source d'une richesse difficilement accessible par d'autres méthodologies. Le fait de rencontrer un individu une vingtaine d'heures, au sein d'un modèle associatif d'entretiens dans lequel la relation interpersonnelle devient tout autant un instrument de connaissances que le discours lui-même, donne au chercheur la possibilité d'effectuer des inférences d'une plus grande validité sur les enjeux inconscients du sujet. Les résultats obtenus ici soutiennent nombre d'éléments présents dans les théories récentes de la dynamique criminelle et laissent croire qu'il est pertinent de poursuivre cette exploration des influences multiples et contradictoires de la figure paternelle car, au-delà de l'importance de la mère, il appert qu'une déception liée à l'image du père puisse, dans certains cas, constituer une motivation importante au surinvestissement du Moi idéal et à l'agir antisocial.

\author{
France Gabrion \\ france.gabrion@cstrois-lacs.qc.ca/ \\ gabrion.france@courrier.uqam.ca
}

Louis Brunet

brunet.louis@uqam.ca

\title{
Note
}

1. Tous les prénoms, de même que les informations qui pourraient permettre l'identification du sujet, sont modifiés afin de protéger son anonymat. De plus, les détails partagés à son propos n'outrepasseront pas ce qui est nécessaire à la compréhension du cas (Gabbard, 2000).

\section{Références}

Aichorn, A., 1925, Wayward Youth, New York, The Viking Press, 1965.

Brunet, L., 2009, La recherche psychanalytique et la recherche sur les thérapeutiques psychanalytiques, Réflexions d'un psychanalyste et chercheur, Filigrane, no ${ }^{\circ}$, 70-85.

Brunet, L., Casoni, D., 2003, Culpabilité, honte et dynamique criminelle, Au sujet des fonctions anti-pulsionnelles et anti-narcissiques du Surmoi, Revue française de psychanalyse, $\mathrm{n}^{\circ} 5$, 1561-1565.

Casoni, D., Brunet, L., 2003, La psychocriminologie, Apports psychanalytiques et applications cliniques, Montréal, Les Presses de l'Université de Montréal.

Casoni, D., Brunet, L., 2007, The psychodynamics that lead to violence, Part 1, The case of the chronically violent delinquent, Canadian Journal of Psychoanalysis, $\mathrm{n}^{\circ}$ 1, 41-55.

Chemama, R., 1995, Dictionnaire de la psychanalyse, Paris, Larousse.

Freud, S., 1900, L'interprétation du rêve, in Euvres complètes, IV, Paris, Presses universitaires de France, 2003. 
Freud, S., 1923, Le Moi et le Ça, in Euvres complètes, XVI, Paris, Presses Universitaires de France, 1991, 255-301.

Freud, A., 1949, Le Moi et les mécanismes de défense, Paris, Presses universitaires de France, 2001.

Gabbard, G., 2000, Disguise or consent: problems and recommendations concerning the publication and presentation of clinical material, The International Journal of Psychoanalysis, $\mathrm{n}^{\circ}$ 6, 1071-1086.

Johnson, A. M., Szurek, S. A., 1952, The genesis of antisocial acting out in children and adults, Psychoanalytic Quarterly, no 3, 323-343.

Kernberg, O., 1975, Borderline Conditions and Pathological Narcissism, New York, Aronson.

Klein, M., 1946, Notes sur quelques mécanismes schizoïde, in Klein M., Heimann, P., Isaacs, S., Rivière, J., éds., Développements de la psychanalyse, Paris, Presses universitaires de France, 1987, 274-300.

Kvale, S., 1999, The Psychoanalytic Interview as Qualitative Research, Qualitative Inquiry, $\mathrm{n}^{\circ}$ 1, 87-113.

Lagache, D., 1951, La psycho-criminogenèse, Revue française de psychanalyse, $\mathrm{n}^{\circ}$ 1, 103-129.

Lagache, D., 1961. La psychanalyse et la structure de la personnalité, in Lagache, D., éd., La psychanalyse, Paris, Presses universitaires de France, 5-55.

Lampl de Groot, J., 1963, Idéal du Moi et Surmoi, Revue française de psychanalyse, $\mathrm{n}^{\text {os }} 4$-5, 529541.

Laplanche, J., Pontalis, J.-B., 1967, Vocabulaire de la psychanalyse, Paris, Presses universitaires de France, 2007.

Lussier, A., 1975, Essai sur l'Idéal du Moi, thèse de doctorat (Ph.D.) sous la direction de Noël Mailloux, Département de psychologie, Université de Montréal.

Lussier, A., 2006, La gloire et la faute. Essai psychanalytique sur le conflit qui oppose narcissisme et culpabilité, Montréal, Presses de l'Université du Québec.

Mailloux, N., 1965, Le fonctionnement du Surmoi chez le délinquant habituel, Contribution à l'étude des sciences de l'homme, $\mathrm{n}^{\circ}$ 6, 67-72.

Mailloux, N., 1971, Jeunes sans dialogue, Paris, Fleurus.

Pedinielli, J.-L., Fernandez, L., 2009, L'observation clinique et l'étude de cas, Barcelone, Armand Colin.

Redl, F., 1945, The Psychology of Gang Formation and the Treatment of Juvenile Delinquents, Psychoanalytic Study of the Child, 1, 367-377.

Sarnoff, I., 1951, Identification with the Aggressor: Some Personality Correlates of Antisemitism Among Jews, Journal of Personality, n 2, 199-218. 